\title{
Mapping clinicopathological entities within colorectal mucinous adenocarcinomas: a hierarchical clustering approach
}

\author{
Charly Liddell ${ }^{1}$, Laure Droy-Dupré ${ }^{1,5}$, Sylvie Métairie ${ }^{2}$, Fabrice Airaud ${ }^{3}$, Christelle Volteau ${ }^{4}$, \\ Stéphane Bezieau ${ }^{3}$, Christian L Laboisse ${ }^{1}$ and Jean-François Mosnier ${ }^{1}$ \\ ${ }^{1}$ Department of Pathology, Hôtel Dieu, Centre Hospitalier Universitaire of Nantes, Nantes, France; \\ ${ }^{2}$ Department of Digestive Surgery, Hôtel Dieu, Centre Hospitalier Universitaire of Nantes, Nantes, France; \\ ${ }^{3}$ Department of Genetics, Hôtel Dieu, Centre Hospitalier Universitaire of Nantes, Nantes, France and \\ ${ }^{4}$ Department of Biostatistics, Hôtel Dieu, Centre Hospitalier Universitaire of Nantes, Nantes, France
}

\begin{abstract}
The aim of this study was to interrogate the heterogeneity of colorectal mucinous adenocarcinomas. This study is based on hierarchical clustering approach combining clinicopathological and molecular patterns known to be relevant to oncogenesis and therapeutic management of patients with colorectal carcinoma, ie, microsatellite instability, 06-methylguanine-DNA methyltransferase (MGMT) status, KRAS, and BRAF mutations and wnt signaling pathway activation. Comparison of the study group of 60 mucinous adenocarcinomas defined according to World Health Organization classification with control group of 136 colorectal adenocarcinomas successively removed shows higher frequency of BRAF and KRAS mutations and microsatellite instability-high status and lower frequency of wnt signaling pathway activation in mucinous adenocarcinomas. Hierarchical clustering isolated three relevant clusters: (i) cluster of microsatellite stable mucinous adenocarcinomas (54\%) with KRAS mutation, and frequent MGMT changes, more frequently located in the left colon, often associated with contiguous precursor adenoma; (ii) cluster of BRAF-mutated mucinous adenocarcinomas $(28 \%)$ with either microsatellite instability-high or microsatellite stable status, occurring in elderly female patients, nearly all located in the right colon, having the signature of serrated pathway of carcinomas; and (iii) a heterogeneous cluster of microsatellite instability-high mucinous carcinomas (18\%), including inherited colorectal carcinomas, displaying a high-grade histological pattern. Age, TNM stage, and BRAF mutation had prognostic value. Hierarchical clustering analysis led to the identification of several clinicopathological entities of colorectal mucinous adenocarcinomas with epidemiologic, prognostic, and therapy relevance. Both KRAS and BRAF mutations appear as drivers in the alternate oncogenetic pathways governing the development of sporadic colorectal mucinous adenocarcinomas.

Modern Pathology (2017) 30, 1177-1189; doi:10.1038/modpathol.2017.18; published online 21 April 2017
\end{abstract}

Mucinous adenocarcinoma is a histological subtype of colorectal carcinomas, first described by Parham in 1923. ${ }^{1}$ According to the World Health Organization definition, ${ }^{2}$ mucinous adenocarcinomas are tumors comprising more than $50 \%$ extracellular mucin. Tumor cells float in mucin pools, often lined by strips of polarized mucin-producing tumor cells. Historically, the main clinicopathological and molecular

Correspondence: Professor J-F Mosnier, MD, PhD, Department of Pathology, Hôtel Dieu, Centre Hospitalier Universitaire of Nantes, 1 place Ricordeau, Nantes F-44093, France.

E-mail: jfmosnier@live.fr

${ }^{5}$ Current address: Service d'Anatomie Pathologique, Hôpitaux Civils de Colmar, F-68024 Colmar, France.

Received 12 September 2016; revised 15 February 2016; accepted 19 February 2017; published online 21 April 2017 characteristics of mucinous adenocarcinomas were inferred from studies aimed at comparing these tumors to non mucinous adenocarcinomas. ${ }^{3-7}$ These studies have led to a seemingly homogeneous representation of mucinous adenocarcinomas, resulting in stereotypes such as location in the right colon, microsatellite instability status, and poor outcome. ${ }^{3-12}$ The aim of the present work is to examine the potential heterogeneity of colorectal mucinous adenocarcinomas on the basis of a hierarchical clustering approach combining clinicopathological and molecular patterns. To this end, besides the classical main clinicopathological parameters of colorectal carcinomas, we introduced in the hierarchical clustering molecular parameters. These include microsatellite instability status, wnt signaling pathway activation, $K R A S$, and $B R A F$ mutational status. In fact, these parameters belong to 
distinct pathways of colorectal oncogenesis ${ }^{13-15}$ and are commonly evaluated in a clinical setting. ${ }^{16,17} \mathrm{We}$ added the evaluation of O6-methylguanine-DNA methyltransferase (MGMT) status in terms of promoter methylation and protein expression, the loss of this enzyme being responsible for deficit repair of alkylation-based DNA alterations. ${ }^{18}$

\section{Materials and methods}

\section{Patients}

Patients diagnosed with primary invasive adenocarcinoma of the colon or rectum showing mucinous differentiation, accessioned at the Department of Pathology at the University Hospital of Nantes, France, between 2004 and 2012 were identified by review of an institutional file. Adenocarcinomas of the rectum treated by neoadjuvant therapy were excluded from this study, as well as the adenocarcinomas of the appendix. A cohort of 136 patients with colorectal adenocarcinomas, not otherwise specified, without any mucinous component and a cohort of 30 patients with colorectal adenocarcinoma with mucinous component accounting for $<50 \%$ of the tumor volume successively removed in our institution served as control groups. Mucinous adenocarcinoma was defined according to 2010 World Health Organization classification $^{2}$ if $>50 \%$ of the lesion is composed of pools of extracellular mucin that contain malignant epithelium as acinar structures, layer of tumor cells, or individual tumor cells including signet ring cells. The pathology reports and hospital charts were reviewed, and the following information was obtained: age, gender, presence of risk factors including colorectal polyposis syndrome and inflammatory bowel disease, type of initial surgical procedure, and the anatomic site of tumor at initial presentation. The investigations were carried out according to the French Ethical Laws. Informed consent was obtained for the collection of specimens declared at the local Ethical Committee under the $\mathrm{n}^{\circ}$ RC_0307.

\section{Pathological Evaluation}

All cases of colorectal adenocarcinoma diagnosed as having mucinous component were histologically reviewed, and the following histological features were recorded for each tumor: percentage of tumor volume composed of extracellular mucin, percentage of acinar pattern, and polarized cell layer pattern defining a high and a low grade of mucinous adenocarcinomas, respectively, and finally the presence or absence of signet ring cells floating in the mucus or poorly cohesive in the desmoplastic stroma. Presence of tumor-infiltrating lymphocytes and Crohn-like reaction defined according to Bethesda guidelines was assessed. ${ }^{19}$ In addition, the extent of invasion, lymph nodes metastases, lymphatic invasion, perineural invasion, extramural venous invasion, presence and type of precursor lesion, and finally synchronous colorectal polyps or colorectal carcinomas were scored. Tumor heterogeneity was considered when two or more distinct histological types, defined according to World Health Organization classification, were distinguished in a same tumor and the percentage of each distinct component was assessed.

\section{Immunohistochemistry}

Three micrometer sections of selected formalin-fixed paraffin-embedded tissue blocks were immunostained with the primary antibodies detailed in Table 1 using peroxydase-labeled polymer method (Envision Flex Plus kit, Dako, Glostrup, Denmark) in a Dako Autostainer after elimination of endogenous peroxydase.

\section{Mismatch Repair and MGMT Proteins}

In tumor cells, MGMT, MLH1, MSH2, MSH6 and PMS2 expression was assessed as retained (normal) or lost. The nuclear expression of MGMT, MLH1, MSH2, MSH6 and PMS2 in intratumoral lymphocytes or peritumoral normal tissue served as positive internal control.

\section{Methylation of MGMT and MLH1 Gene Promoter}

MLH1 and MGMT promoter methylation was determined using MethyLight technique. Tumor DNA was extracted from paraffin sections using iPrep TM Charge Switch Forensic in automated iPrep TM

Table 1 Antibodies and dilutions used

\begin{tabular}{lcl}
\hline Antibodies & Dilution & Source \\
\hline MGMT (clone MT3.1) & $1: 100$ & Labvision, Fremont, CA, USA \\
Beta-catenin (clone CAT 5H10) & $1: 100$ & Invitrogen, Carlsbad, CA, USA \\
MLH1 (clone G168-15) & $1: 100$ & BD Biosciences, Erembodegem, Belgium \\
MSH2 (clone D06578-8) & $1: 100$ & Oncogene, Cambridge, MA, USA \\
MSH6 (clone 44) & $1: 40$ & BD Transduction Laboratories, Lexington, KY, USA \\
PMS2 (clone EP51) & Prediluted & Dako, Glostrup, Denmark \\
\hline
\end{tabular}

Abbreviation: MGMT, Methyl Guanine-DNA Methyl Transferase. 
Purification instrument (Invitrogen, Carlsbad, CA, USA). Genomic DNA was then treated with bisulfite using Epitect Bisulfite kit (Qiagen, Hilden, Germany). Primer sequences of MLH1 promoter for the methylated reaction were 5'-AGGAAGAGCGGATA GCGATTT-3' (sense) and 5'-TCTTCGTCCCTCCCTA AAACG-3' (antisense). Primer sequences of MLH1 promoter for the methylated reaction were $5^{\prime}$ - TGTG TTTTAAGGGGAAGATGG-3' (sense) and 5'-CCAA TACCTATCCCCACCTC-3' (antisense). The CoL2A1 control reaction was used to normalize for bisulfiteconverted input DNA. The loci were considered to be methylated if the percentage of methylated reference was higher than $10 .^{20}$

\section{Microsatellite Instability Status}

For determining microsatellite instability status, DNA was extracted from $10 \mu \mathrm{m}$-thick sections. The microsatellite instability status was determined by PCR, by amplifying the five mononucleotide markers BAT25, BAT26, NR21, NR22 and NR24, designed and validated previously. ${ }^{21}$

\section{Assessment of Nuclear Beta-Catenin Expression}

Immunohistochemistry for beta-catenin was assessed as surrogate marker for wnt/beta-catenin signaling pathway activation in mucinous adenocarcinomas and controls. The number of nuclei immunostained for beta-catenin was quantified in the center of mucinous carcinomas in three different fields at $\times 400$ magnification, representing the areas the most enriched in beta-catenin-positive nuclei scanned at a low magnification. The beta-cateninpositive nuclei in the tumor cells were then counted with the NE Elements Imaging Software (Nikon, Edo, Japan).

\section{Mutational Analysis of CTNNB1}

In MSI-H colorectal mucinous adenocarcinomas, exon 3 of CTNNB1 gene was amplified by PCR using the following primers: forward 5'-TGTAAAACG ACGGCCAGTGAACCAGACAGAAAAGCGGCTG-3' and reverse 5'-CAGGAAACGGCTATGACCACTCAT ACAGGACTTGGGAGG-3' ${ }^{22}$ Sanger sequencing of PCR products was performed using Big Dye Terminator v1.1 cycle sequencing kit (Thermo Fisher Scientific) and run on a ABI prism 3130XL automatic sequencer (Applied Biosystems).

\section{Mutational Analysis of $B R A F$ and $K R A S$}

Analysis of $B R A F$ and KRAS was performed by automated sequencing. The fragments encompassing exon 15 of gene $B R A F$ and exon 2, 3, and 4 of gene KRAS were amplified by PCR. PCR products were purified and sequenced on a ABI Prism 3130XL automatic sequencer (Applied Biosystems) using the Seqscape software (Applied Biosystems).

\section{Hierarchical Clustering Analysis of Mucinous Adenocarcinomas}

Data were specifically reformatted for the hierarchical clustering analysis. For mutation of $B R A F$ and $K R A S$, microsatellite instability, a three-point scale was used where $0=$ no mutation or no microsatellite instability; 1 = uninterpretable, and $3=$ mutation or microsatellite instability. For nuclear beta-catenin expression, a four-point scale was used where $0=0-$ $<10 \%$ of positive nuclei, $1=$ uninterpretable, $2=\geq 10-<50 \%$ of positive nuclei, and $3=\geq 50 \%$ of positive nuclei. For MLH1, MSH2, and PMS2 expressions, a three-point scale was used where $0=$ normal expression (positive), 1 = uninterpretable, and $3=$ loss of expression (negative). Finally for MGMT, a three-point scale was used where $0=$ normal expression and no MGMT methylation promoter, $1=$ uninterpretable, and $3=$ either loss of expression and/or MGMT promoter methylation. Hierarchical clustering analysis was performed using Cluster and Tree View software, freely available at http://rana.stanford.edu/software/.23,24

\section{Statistics}

Associations between categorical variables (cluster of differentiation, MGMT status, gender, microsatellite instability status, tumor location) were calculated using $\chi^{2}$-test, Fisher's exact test, or Kruskal-Wallis test as appropriate. Mann-Whitney test was used to compare the continuous variables between groups.

Intraoperative and clinical follow-up data were obtained from hospital charts. Disease-specific survival was measured from the date of surgery to the date of death related to colonic carcinoma (ie, death unrelated to colonic carcinoma was censored at the time of death). Univariate survival analysis was performed using the log-rank test and illustrated using Kaplan-Meier Curves. $P$-values $<0.05$ were considered statistically significant. Statistical analysis was performed using SAS software version 9.3 for Windows (SAS Institute, Cary, NC, USA).

\section{Results}

\section{Histological Analysis of Mucinous Adenocarcinomas}

A total of 845 primary colorectal carcinomas were surgically removed from 2004 to 2012. Of these, 60 $(7.1 \%)$ could be considered as mucinous adenocarcinomas according to the criteria of 2010 World Health Organization classification. ${ }^{2}$ As described in the World Health Organization classification, mucinous adenocarcinomas were further subdivided 

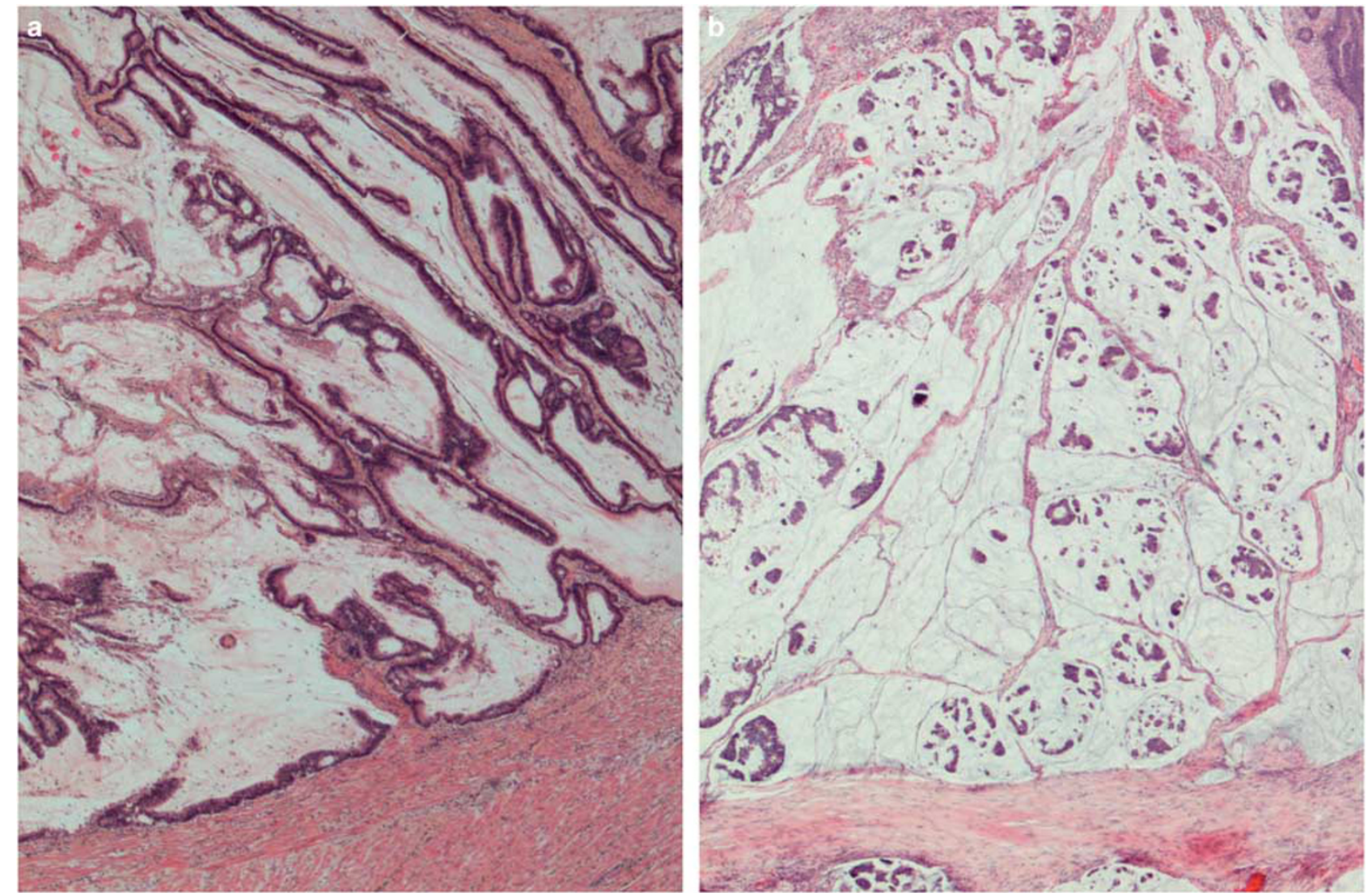

Figure 1 (a) Low-grade mucinous adenocarcinoma characterized by polarized epithelial cell layers lining pools of mucin. (b) Highgrade mucinous adenocarcinoma characterized by acinar structures floating in pools of mucin (hematein-eosin, original magnification $\times 40)$.

into two groups according to their architectural phenotype: (i) mucinous adenocarcinomas consisting in polarized epithelial cell layers lining pools of mucin (low-grade mucinous adenocarcinoma: $n=37$, $62 \%$ ) (Figure 1a); and (ii) mucinous adenocarcinomas with acinar structures floating within pools of mucin (high-grade mucinous adenocarcinoma: $n=23,38 \%$ ) (Figure 1b). Forty nine mucinous adenocarcinomas were entirely composed of extracellular pools of mucin, whereas 11 cases had variable proportion of extracellular mucin (between 50 and $90 \%$ ). Finally, a signet ring cell component was observed in 13 mucinous adenocarcinomas (Figure 2). Signet ring cells were more frequently detected in the mucinous adenocarcinomas with acinar structures floating within extracellular pools of mucin than in mucinous adenocarcinomas with polarized epithelial cell layers lining pools of mucin (10/23 (43\%) vs 3/37 (8\%) $\left(\chi^{2}\right.$-test: $\left.P=0.001\right)$. Furthermore, component of signet ring cells with infiltrative growth pattern in desmoplastic stroma was present in 6 of these 13 cases (46\%). Tumor-infiltrating lymphocytes were detected in 11 mucinous adenocarcinomas (18\%) and Crohn-like reaction in 12 mucinous adenocarcinomas $(20 \%)$.

\section{Nuclear Beta-Catenin Expression}

The rate of nuclear expression of beta-catenin was assessed to evaluate the activation level of wnt signaling pathway in the tumor core. Beta-catenin was expressed in 0-92\% of tumor cell nuclei (mean number $=21 \%)$. Only two cases had no nuclei $(0 \%)$ immunostained for beta-catenin. The mean number of tumor cells with nuclear beta-catenin expression was lower in mucinous adenocarcinoma than in the control group of adenocarcinomas, not other specified $(21 \%$ vs $30 \%$; Mann-Whitney test, $P=0.002)$ and slightly higher than in the control group of adenocarcinoma with mucinous component (21\% vs $16 \%, P=0.891$ ) (Table 2).

\section{KRAS Mutational Status}

KRAS exon 2 mutation was detected in 24 of the 60 mucinous adenocarcinomas (40\%). Although the KRAS mutation rate was higher in mucinous adenocarcinomas than in the control group of adenocarcinomas, not other specified $(45 / 136 ; 33 \%)$, the difference was not statistically significant. KRAS mutations were as frequent in mucinous carcinomas 


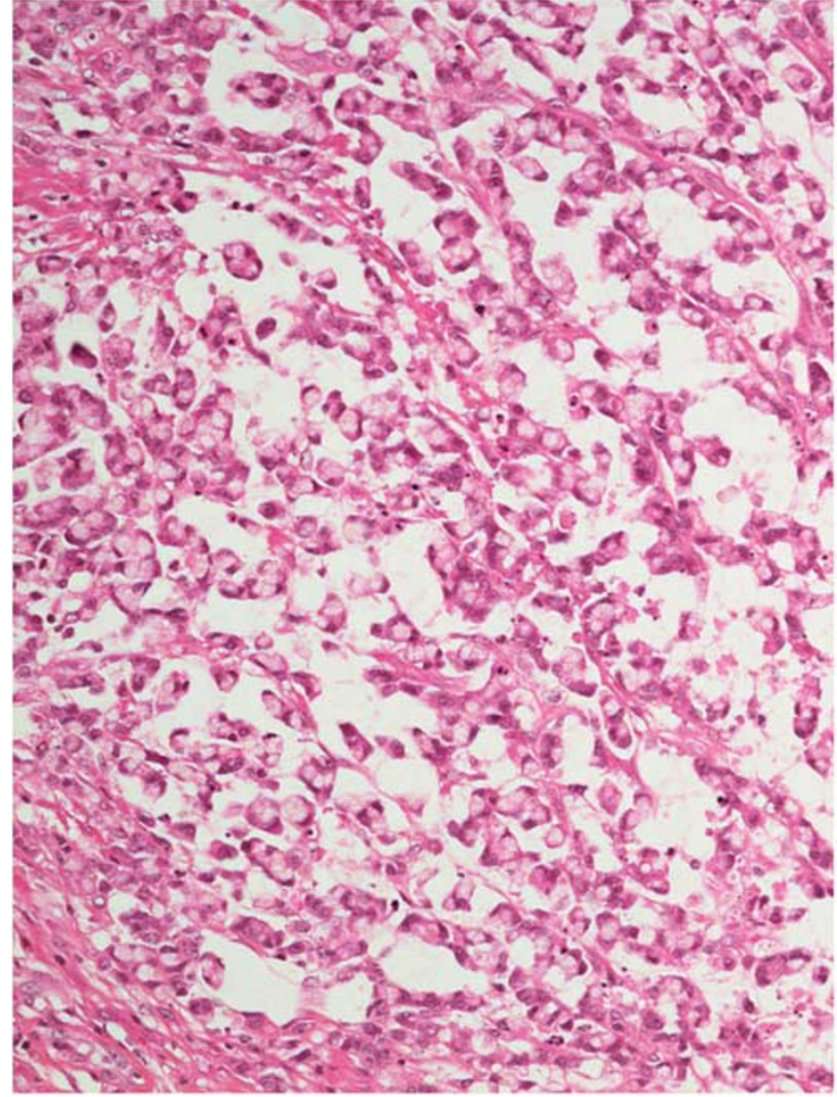

Figure 2 Presence of signet ring cells in pools of mucin (hemateineosin, original magnification $\times 200$ ).

adenocarcinomas as in adenocarcinomas with mucinous component $(12 / 30,40 \%)$.

\section{BRAF Mutational Status}

Seventeen (28\%) mucinous carcinomas harbored $B R A F$ mutation. All of these mutations were the driver mutation V600E but one (G596R). The rate of BRAF mutation in mucinous carcinomas was significantly higher than in the control group of adenocarcinomas, not other specified $(4 / 136 ; 3 \%)\left(\chi^{2}\right.$-test: $\left.P<0.0001\right)$. The rate of BRAF mutation was higher in mucinous carcinomas than in adenocarcinomas with mucinous component but the difference was not statistically significant (28\% vs $17 \%$; Fisher exact test: $P=0.226$ ).

\section{Microsatellite Instability Status}

Twenty of the $60(33 \%)$ mucinous adenocarcinomas showed high degree of microsatellite instability: 17 with concurrent loss of MLH1 and PMS2 expression at the immunohistochemical level, 2 with concurrent loss of MSH2 and MSH6 immunohistochemical expression, and 1 with isolated loss of PMS2 immunohistochemical expression. Of the 20 mucinous adenocarcinomas with high degree of microsatellite instability, 9 (45\%) were positive for $B R A F$ mutation. Microsatellite instability was associated to a proved Lynch syndrome in two patients with mucinous carcinomas. MLH1 methylation was detected in 18 cases, including 10 BRAFmutated mucinous adenocarcinomas and 1 mucinous adenocarcinoma associated to Lynch syndrome.

In the control group of adenocarcinomas, not other specified, 5 of the $136(4 \%)$ tumors showed high degree of microsatellite instability ( $\chi^{2}$-test: $P<0.0001$ ), whereas in the control group of adenocarcinomas with mucinous component, 10 of the 30 tumors $(33 \%)$ showed high degree of microsatellite instability $\left(\chi^{2}\right.$-test, $\left.P=1\right)$.

\section{MGMT Promoter Methylation and Loss of MGMT Expression}

MGMT hypermethylation was found in 17 of the 60 mucinous adenocarcinomas $(28 \%)$, whereas immunohistochemical loss of MGMT expression was observed in 14 adenocarcinomas (23\%) (Figure 3). MGMT promoter methylation was highly correlated with loss of MGMT expression $(P<0.001)$ but they did not match perfectly. A loss of MGMT expression was observed in three cases with no MGMT promoter methylation and the MGMT expression was conserved in six tumors with MGMT methylation. Finally a total of 20 mucinous carcinomas were associated with MGMT changes, ie, either a loss of MGMT expression or MGMT promoter hypermethylation. The frequency of KRAS G>A mutation was significantly higher in mucinous adenocarcinomas with immunohistochemical loss of MGMT expression $(7 / 14,50 \%)$ than in mucinous adenocarcinomas with MGMT expression (8/46, 17\%, $P=0.013)$.

\section{Clinical Outcome}

Follow-up data were available for 58 of the 60 patients with mucinous adenocarcinomas. The mean duration of follow-up was 34 months (1-105 months). The prognostic significance of (i) each single molecular parameter, (ii) demographic and clinicopathological parameter, including microsatellite instability status was determined using univariate and multivariate statistical analysis.

In the univariate analysis, only age of patients at the diagnosis had a prognostic value (HR 1.05, 95\% CI 1.02-1.09, $P=0.0033$ ). The prognostic value of $B R A F$ mutation exhibited a tendency toward significance (HR 1.97, 95\% CI 0.92-4.23, $P=0.0754$ ). In the multivariate survival analysis using Cox's regression model, age at the diagnosis, BRAF mutation, and TNM stage 4 had an independent negative association with overall survival (Table 3).

\section{Hierarchical Clustering of Mucinous Adenocarcinomas}

The hierarchical clustering analysis was based on molecular data described previously, ie, nuclear beta-catenin expression, KRAS and BRAF mutational 
Table 2 Comparison of the clinicopathological and molecular findings between the study group of mucinous adenocarcinomas and the control groups of adenocarcinoma, no other specified and adenocarcinoma with mucinous component $<50 \%$

\begin{tabular}{|c|c|c|c|c|c|}
\hline & $\begin{array}{l}\text { Mucinous } \\
\text { carcinomas }\end{array}$ & Adenocarcinomas & $\mathrm{P}$-value ${ }^{\mathrm{a}}$ & $\begin{array}{l}\text { Adenocarcinomas with } \\
\text { mucinous component }\end{array}$ & P-value \\
\hline$N(\%)$ & 60 & 136 & & 30 & \\
\hline Gender & & & NS & & NS \\
\hline Male & $32(62.5 \%)$ & $81(63 \%)$ & & $15(50 \%)$ & \\
\hline Female & $28(37.5 \%)$ & $55(37 \%)$ & & $15(50 \%)$ & \\
\hline Mean age (years) & 70 & 68 & $0.456^{\mathrm{b}}$ & 72 & $0.312^{\mathrm{b}}$ \\
\hline$<55$ years & $10(17 \%)$ & $19(14 \%)$ & & $2(7 \%)$ & \\
\hline $56-75$ years & $24(40 \%)$ & $70(51 \%)$ & & $16(53 \%)$ & \\
\hline$>75$ years & $26(43 \%)$ & $47(35 \%)$ & & $12(40 \%)$ & \\
\hline Location & & & $<0.0001$ & & 0.648 \\
\hline Right colon & $37(62 \%)$ & $39(29 \%)$ & & $17(57 \%)$ & \\
\hline Left colon & $23(36 \%)$ & $97(71 \%)$ & & $13(43 \%)$ & \\
\hline Precursor adenomas & $20(33 \%)$ & $26(19 \%)$ & 0.03 & $7(23 \%)$ & 0.09 \\
\hline Conventional & $16(27 \%)$ & $26(19 \%)$ & & $6(20 \%)$ & \\
\hline Serrated & $4(6 \%)$ & $0(0 \%)$ & & $1(3 \%)$ & \\
\hline Associated carcinoma & $10(17 \%)$ & $16(12 \%)$ & 0.351 & $2(7 \%)$ & 0.188 \\
\hline Colorectal & $6(10 \%)$ & $9(7 \%)$ & & 0 & \\
\hline Other organs & $4(6 \%)$ & $7(5 \%)$ & & $2(7 \%)$ & \\
\hline Inflammatory bowel disease & 3 & 0 & ND & 0 & ND \\
\hline BRAF mutation & $17(28 \%)$ & $4(3 \%)$ & $<0.0001$ & $5(17 \%)$ & 0.224 \\
\hline KRAS mutation & $24(40 \%)$ & $45(33 \%)$ & 0.350 & $12(40 \%)$ & 1 \\
\hline MSI-H & $20(30 \%)$ & $5(4 \%)$ & $<0.0001$ & $10(33 \%)$ & 1 \\
\hline $\begin{array}{l}\text { Mean nuclear beta-catenin } \\
\text { expression }\end{array}$ & $21 \%$ & $30 \%$ & $0.002^{\mathrm{C}}$ & $16 \%$ & 0.891 \\
\hline
\end{tabular}

Abbreviation: ND, not done.

${ }^{a}$ All the statistics: $\chi^{2}$ - or Fisher tests unless.

${ }^{\mathrm{b}}$ Kruskal-Wallis test.

${ }^{\mathrm{C}}$ Mann-Whitney test

status, microsatellite instability status, loss of MSH2 expression, and MGMT changes. The hierarchical clustering allows dividing mucinous adenocarcinomas into four clusters as shown in Figure 4. The first cluster (C1) consisted of 24 mucinous adenocarcinomas with KRAS mutation and microsatellite stable status. In this cluster, MGMT changes were frequent (13 of 24 (54\%); $P=0.01$ vs other clusters). These mucinous carcinomas were often located in the left side $(14 / 24(59 \%))$ and were frequently combined with detectable precursor lesions such as advanced villous adenomas, tubulo-villous adenomas, or even traditional serrated adenomas. As for all the other clinicopathological features, the rate of nuclear betacatenin expression was not different to that of the control group of adenocarcinomas, not other specified. The second cluster (C2) consisted of $17 B R A F$ mutated mucinous carcinomas. The number of elderly patients, essentially females, was higher in this cluster (Table 4). Notably, this cluster comprised as many microsatellite instability-high mucinous carcinomas (9/17) as microsatellite stable mucinous carcinomas (8/17). However the rate of nuclear betacatenin expression was significantly lower than those of all other clusters (Mann-Whitney test, $P<0.0001)$ as well as of that of control group of adenocarcinoma, not other specified $(P=0.002)$.
Some of the $\mathrm{C} 2$ mucinous adenocarcinomas harbored MGMT changes. Finally, according to the results of the clinical outcome study, this cluster had the worse prognosis. The third cluster (C3) consisted of 11 microsatellite instability-high mucinous carcinomas, termed as 'microsatellite instabilityhigh null' because KRAS and BRAF mutations were lacking. Some of their clinicopathological findings were significantly different. All the cases were located in the right colon (C3 vs others, Fisher exact test, $P=0.01$ ) and most exhibited histological patterns associated with high grade of differentiation (C3 vs others, Fisher exact test $P=0.001$ ), including signet ring cell floating in mucin pools $(P=0.002)$ and component of signet ring cells with infiltrating growth pattern $(P=0.007)$. This cluster was also more frequently associated with Crohn-like reaction (Fisher exact test; $P=0.01$ ). Tumors-infiltrating lymphocytes were as frequent in this cluster as in BRAFmutated mucinous adenocarcinoma cluster, but significantly more frequent than in clusters C1 and C4. The patients with Lynch syndrome were included in this cluster. In this cluster, the rate of nuclear beta-catenin was lower than in cluster C1 and C4, but significantly higher than in cluster C2 (Mann-Whitney test, $P=0.001$ ). Notably, betacatenin exon 3 mutations (pSer45del and pSer45Phe) 

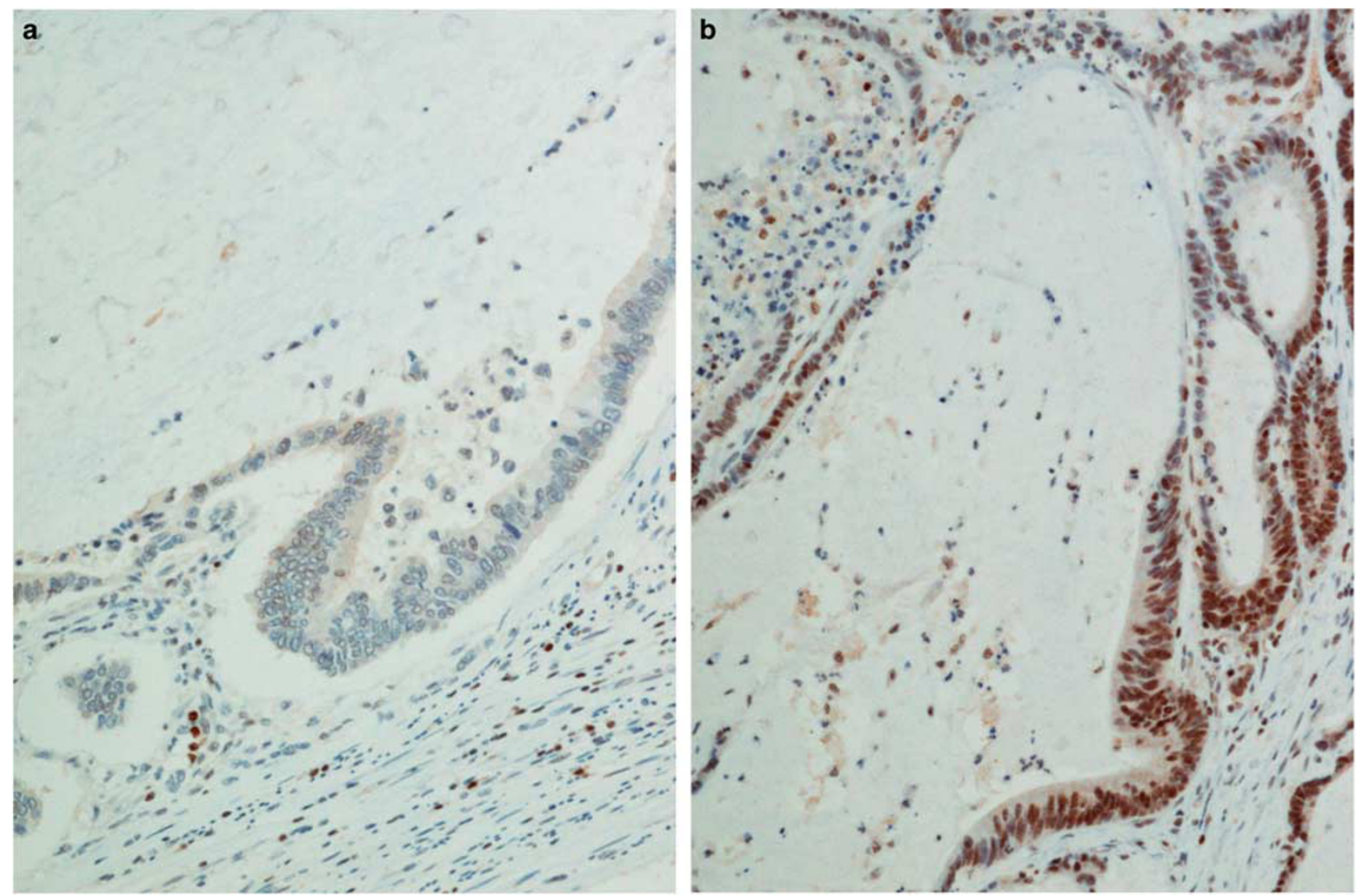

Figure 3 Representative view of MGMT immunostaining. (a) Complete loss of MGMT expression by tumor cells of a mucinous adenocarcinoma. The nuclear expression of MGMT by stromal and inflammatory cells served as internal positive control. (b) Nuclear and cytoplasmic expression of MGMT in nearly all tumor cells of a mucinous adenocarcinoma. (MGMT immunostaining × 200). MGMT, O6-methylguanine-DNA methyltransferase.

Table 3 Impact of clinicopathological characteristics, genotypical markers of oncogenesis, and tumor stage on the disease-specific survival of patients with mucinous adenocarcinomas using multivariate Cox's regression model

\begin{tabular}{lccr}
\hline Variable $^{\mathrm{a}}$ & HR & IC & P-value \\
\hline Age & 1.07 & $1.03-1.11$ & $<0.001$ \\
BRAF mutation & 3.49 & $1.20-10.2$ & 0.021 \\
Signet ring cell & 0.60 & $0.18-1.94$ & 0.389 \\
Acinar pattern & 2.65 & $0.99-7.12$ & 0.053 \\
KRAS mutation & 0.53 & $0.15-1.84$ & 0.317 \\
MLH1 methylation & 0.26 & $0.06-1.21$ & 0.085 \\
MSI/MSS status & 3.09 & $0.63-15.3$ & 0.165 \\
Stage 3 vs 1/2 & 0.20 & $0.04-0.98$ & 0.044 \\
Stage 4 vs 1/2 & 1.67 & $0.55-5.07$ & \\
\hline
\end{tabular}

${ }^{\mathrm{a}}$ Number of cases $=58$.

were detected in mucinous carcinomas of the two patients with proved Lynch syndrome. In these two cases, beta-catenin was expressed in 20 and $35 \%$ of tumor cell nuclei. No MGMT change was recorded in this cluster. MLH1 promoter methylation was present in 8 of the 11 cases of this cluster. As shown in Figure 5, this cluster was probably the most heterogeneous clinically. The fourth cluster (C4) consisted of eight mucinous carcinomas and was termed 'microsatellite stable null' because KRAS and $B R A F$ mutations were lacking. Although this cluster shared many features with cluster C1, MGMT changes were less frequent. The rate of nuclear beta-catenin expression was not different to those of cluster $\mathrm{C} 1$ and control group of adenocarcinomas, not other specified (31\% vs $32 \%$ and $31 \%$ vs $30 \%$, respectively, Mann-Whitney test; $P=$ NS). Globally, this cluster did not show significant difference with the control group of adenocarcinomas, not other specified, except mucinous histological pattern.

A hierarchical clustering analysis of adenocarcinomas with mucinous component $<50 \%$ was undertaken (see Supplementary figure). Adenocarcinomas with mucinous component can be divided into three groups: microsatellite stable, KRAS-mutated $(n=11$; $36 \%)$, microsatellite stable null $(n=9,31 \%)$, and microsatellite instability-high carcinomas $(n=10$; $33 \%)$. In comparison with clusters of mucinous adenocarcinomas, there were some differences: all the $B R A F$-mutated carcinomas fall in microsatellite instability-high tumors and BRAF wild type, microsatellite instability-high cluster included one tumor harboring KRAS mutation and MLH1 promoter methylation associated with conventional villous adenoma $(25 \%)$. 


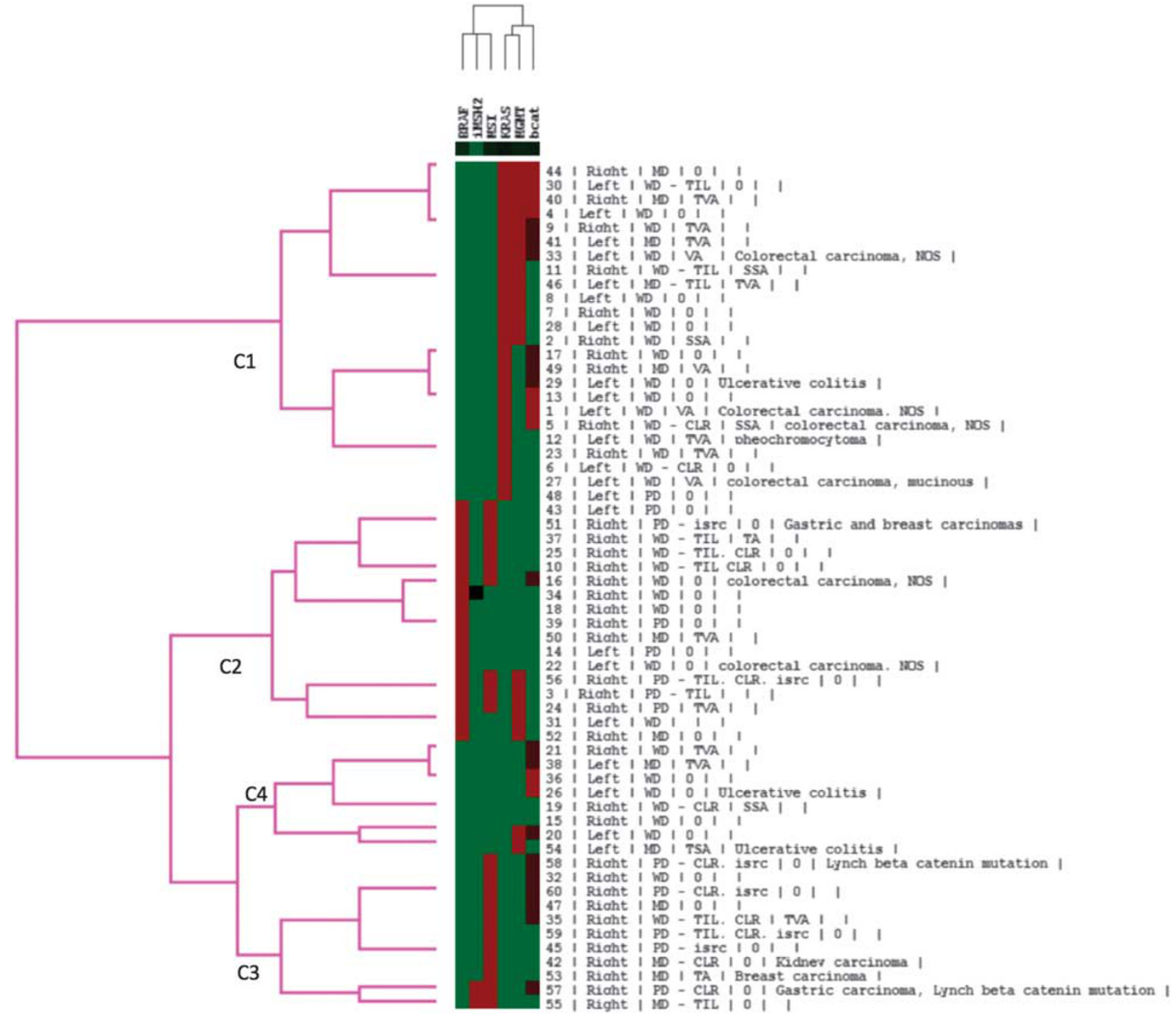

Figure 4 Hierarchical clustering analysis of 60 colonic mucinous adenocarcinomas. In a first step, the tumors are grouped on the basis of the relatedness of KRAS and BRAF mutational status, nuclear beta-catenin expression, microsatellite instability status, MGMT changes (either loss of MGMT expression or methylation of MGMT promoter gene), and loss of MSH2 expression. Then, the patients are sorted out according to their degree of relatedness in the selected profile. The dendograms show two dimensions of clustering, ie, the association between the biomarkers (top) and the association between the patients (left hand side). KRAS mutation, BRAF mutation, microsatellite instability status, MGMT changes, nuclear beta-catenin expression $\geq 50 \%$ of cells in tumor core, and loss of MSH2 expression are represented as bright red blocks; nuclear beta-catenin expression comprised between $\geq 10 \%$ and $<50 \%$ is represented as dark red blocks; no KRAS mutation, no BRAF mutation, microsatellite stable status, no MGMT change, nuclear beta-catenin expression $<10 \%$ in cells of tumor core, and MSH2 expression are represented as green blocks. The look-up in the right side of the figure details some clinicopathological features of the tumors: first column, identification of the studied cases; second column, location of tumor; third column, histological subtype of tumor; fourth column, contiguous precursor polyp if any, fifth column: associated colonic or extra-colonic carcinomas, inflammatory bowel disease or proved inherited colorectal cancer syndrome if any. Four clusters of tumors were hence selected, namely cluster 1 as KRAS-mutated mucinous adenocarcinomas, cluster 2 as BRAF-mutated mucinous adenocarcinomas, cluster 3 as 'microsatellite instability-high null' mucinous adenocarcinomas, and Cluster 4 as 'microsatellite stable null' mucinous adenocarcinomas. CLR, Crohn-like reaction; isrc, infiltrating growth pattern of signet ring cells; MD, acinar pattern or high grade; MGMT, O6-methylguanine-DNA methyltransferase; MSI, microsatellite instability; MSS, microsatellite stable; NOS, no other specified; PD, signet ring cells component; SSA, sessile serrated adenoma; TA, tubulous adenoma; TIL, tumor-infiltrating lymphocytes; TSA, traditional serrated adenoma; TVA, tubulo-villous adenomas; VA, villous adenoma; WD, polarized epithelial cells layer or low grade.

\section{Discussion}

Our understanding of colorectal mucinous adenocarcinomas is historically based on two approaches. First, a descriptive pathological approach has identified some clinicopathological characteristics. ${ }^{3,4}$
Second, the availability of molecular techniques allowed reconsidering the colorectal mucinous adenocarcinomas based on molecularly defined characteristics. ${ }^{5-7,9,10}$ Here we present a convergent approach based on hierarchical clustering that integrates both clinicopathological and molecular 
Table 4 Association of the main clinicopathological features across the four clusters of mucinous adenocarcinomas defined by hierarchical clustering

\begin{tabular}{|c|c|c|c|c|c|}
\hline \multirow{2}{*}{ Clinicopathological parameters } & \multicolumn{4}{|c|}{ Clusters } & \multirow{2}{*}{$\mathrm{P}$-value ${ }^{\mathrm{a}}$} \\
\hline & Cluster 1 & Cluster 2 & Cluster 3 & Cluster 4 & \\
\hline \multirow{4}{*}{$\begin{array}{l}N(\%) \\
\text { Gender } \\
\quad \text { Male } \\
\text { Female }\end{array}$} & $24(40 \%)$ & $17(28 \%)$ & $11(18 \%)$ & $8(13 \%)$ & \multirow{4}{*}{ NS } \\
\hline & & & & & \\
\hline & $15(62.5)$ & $6(35 \%)$ & $6(55 \%)$ & $5(63 \%)$ & \\
\hline & $9(37.5)$ & $11(65 \%)$ & $5(45 \%)$ & $3(37 \%)$ & \\
\hline Mean age (years) & 66 & 76 & 69 & 71 & $0.05^{\mathrm{b}}$ \\
\hline$<55$ years & $6(25 \%)$ & $2(12 \%)$ & $2(18 \%)$ & $0(0 \%)$ & $0.06^{\mathrm{a}}$ \\
\hline $56-75$ years & $10(42 \%)$ & $4(24 \%)$ & $4(36 \%)$ & $6(75 \%)$ & \\
\hline$>75$ years & $8(33 \%)$ & $11(64 \%)$ & $5(46 \%)$ & $2(25 \%)$ & \\
\hline \multicolumn{5}{|l|}{ Location } & \multirow[b]{3}{*}{ C1+C4 vs C2+C3: 0.0004} \\
\hline Right colon & $10(42 \%)$ & $13(76 \%)$ & $11(100 \%)$ & $3(37 \%)$ & \\
\hline Left colon & $14(58 \%)$ & $4(24 \%)$ & $0(0 \%)$ & $5(63 \%)$ & \\
\hline \multicolumn{5}{|l|}{ Tumor grade } & \multirow[t]{3}{*}{ C3 vs other: 0.001} \\
\hline Low grade & $18(75 \%)$ & $11(65 \%)$ & $2(18 \%)$ & $6(75 \%)$ & \\
\hline High grade & $6(25 \%)$ & $6(35 \%)$ & $9(82 \%)$ & $2(25 \%)$ & \\
\hline Prior adenomas & $11(46 \%)$ & $3(18 \%)$ & $2(18 \%)$ & $4(50 \%)$ & NS \\
\hline Conventional & 9 & 3 & 2 & 2 & \\
\hline Serrated & 2 & 0 & 0 & 2 & C1+C4 vs C2+C3: 0.02 \\
\hline Associated carcinoma & $4(17 \%)$ & $3(18 \%)$ & $3(27 \%)$ & $0(0 \%)$ & NS \\
\hline Colorectal & 4 & 2 & 0 & 0 & \\
\hline Extra-colonic & 0 & 1 & 3 & 0 & \\
\hline Inflammatory bowel disease & 2 & 0 & 0 & 1 & ND \\
\hline Lymph node metastases & $13(54 \%)$ & $8(47 \%)$ & $3(27 \%)$ & $4(50 \%)$ & NS \\
\hline MGMT changes & $13(54 \%)$ & $5(29 \%)$ & $0(0 \%)$ & $2(25 \%)$ & C1 vs others: 0.01 \\
\hline Mean nuclear beta-catenin expression & $32 \%$ & $4 \%$ & $15 \%$ & $31 \%$ & C2 vs others: $0.0002^{\mathrm{d}}$ \\
\hline
\end{tabular}

Abbreviation: ND: not done.

${ }^{a}$ All the statistics: $\chi^{2}$ - or Fisher tests unless.

bruskal-Wallis test.

${ }^{\mathrm{c}} \mathrm{C}$ represents cluster.

dMann-Whitney test.

characteristics that underlines the heterogeneity of this group.

Basically, our approach based on hierarchical clustering analysis of colorectal mucinous adenocarcinomas exploited microsatellite instability status, $B R A F$ and KRAS mutational status, and WNT/betacatenin signaling activation. MGMT changes were integrated in this hierarchical clustering analysis because of its known involvement in colorectal oncogenesis through its role as a tumor suppressor function. ${ }^{25,26}$

In a first step, in comparison with a control group of colorectal adenocarcinomas, not other specified, colorectal mucinous adenocarcinomas showed four distinctive major molecular features: (i) significant higher frequency of $B R A F$ mutation, (ii) significant higher frequency of high level of microsatellite instability, (iii) slightly higher frequency of KRAS mutation, and (iv) significant lower wnt/beta-catenin signaling activation in the tumor core. Globally, nearly $70 \%$ of colorectal mucinous adenocarcinomas had a constitutional activation of RAS/RAF/MAP kinase pathway, suggesting that this pathway plays a role in the development of mucinous adenocarcinomas. In the present series, KRAS mutation was the molecular abnormality most frequently detected in mucinous adenocarcinomas. The percentage of colonic mucinous adenocarcinomas in our cohort $(7.4 \%)$ was in the lower range of incidences reported in western countries $(7-19 \%) .{ }^{27-29}$ The clinicopathological and molecular findings of colorectal mucinous adenocarcinomas included in the present series were similar to those of the series recently reported by Andrici et al, ${ }^{30}$ including 264 patients with mucinous adenocarcinomas in order to evaluate the prognostic value of microsatellite instability-high status. However, in the present study, the rate of males was slightly higher than the rate of females and the ratio of colorectal mucinous adenocarcinomas harboring BRAF mutation and high level of microsatellite instability were slightly lower $(28 \%$ vs $35 \%$ and $33 \%$ vs $36 \%$, 


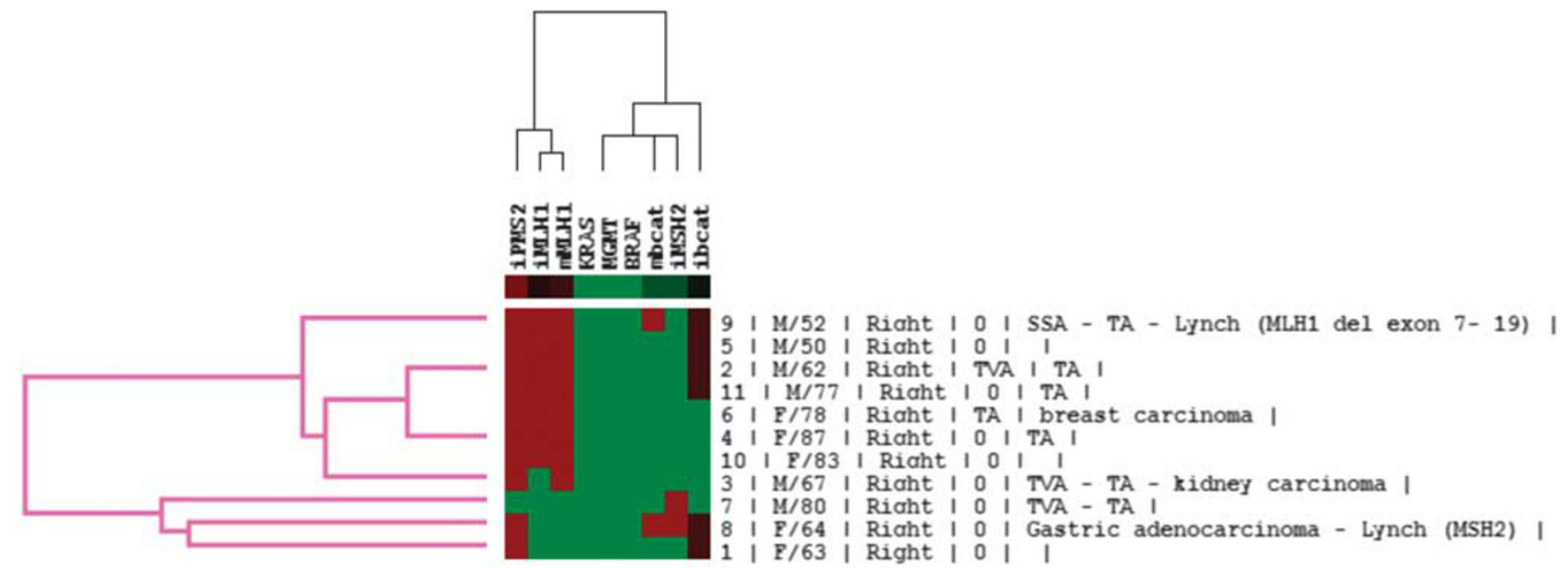

Figure 5 Hierarchical clustering analysis of 'microsatellite instability-high null' mucinous adenocarcinomas. The tumors are grouped on the basis of the relatedness of KRAS and BRAF mutational status, nuclear beta-catenin expression, MGMT changes, MLH1 promoter gene methylation and MLH1, MSH2, or PMS2 expression. Then, the patients are sorted out according to their degree of relatedness in the selected profile. The dendograms show two dimensions of clustering, ie, the association between the biomarkers (top) and the association between the patients (left hand side). KRAS mutation, BRAF mutation, MGMT changes, MLH1 promoter gene methylation, nuclear betacatenin expression $\geq 50 \%$ of cells in tumor core and loss of MLH1, MSH2, or PMS2 expression are represented as bright red blocks; nuclear beta-catenin expression comprised between $\geq 10 \%$ and $<50 \%$ is represented as dark red blocks; no KRAS mutation, no BRAF mutation, no MLH1 promoter gene methylation, no MGMT change, nuclear beta-catenin expression $<10 \%$ in cells of tumor core and MLH1, MSH2, or PMS2 expression are represented as green blocks. The look-up in the right side of the figure details some clinicopathological features of the patients: first column, identification of the studied case; second column, gender and age at diagnosis of patients; third column: location of tumor; fourth column, contiguous precursor polyp if any, fifth column: associated colonic or extracolonic carcinomas and proved inherited colorectal cancer syndrome if any. 'microsatellite instability-high null' cluster appears clinically heterogeneous including patients with proved Lynch syndrome, patients with unproved suspected Lynch syndrome, patients with Lynch-like syndrome and unproved sporadic microsatellite instability-high serrated pathway-associated mucinous carcinomas. MGMT, O6-methylguanine-DNA methyltransferase.

respectively). Notably, Andrici et al ${ }^{30}$ only used immunohistochemistry, applied on tissue microarrays, to determine both $B R A F$ mutational status and microsatellite instability status. Unfortunately, these authors did not report KRAS mutational status of their large series of mucinous adenocarcinomas. However, other series including any histological subtypes of colorectal adenocarcinomas have reported that KRAS mutation was more frequent in colorectal adenocarcinomas with extracellular mucinous component. ${ }^{31,32}$

From a histological point of view, this study shows that mucinous component per se, whatever its volume, has no definite value for determining molecular profile of colorectal carcinomas. However the combination of patterns such as growth pattern of mucinous component, presence of signet ring cells floating in mucin pools, component of signet ring cells infiltrating growth pattern, residual polyps at the edge of tumor, tumor-infiltrating lymphocytes, and Crohnlike reaction can help predict molecular clusters of colorectal carcinomas. These findings should be taken into account when establishing the histomolecular classification of colorectal carcinomas.

Hierarchical clustering analysis identified four main clusters of colorectal mucinous adenocarcinomas, which can be broadly defined as (i) $B R A F$-mutated mucinous adenocarcinomas, (ii) 'microsatellite instability—high null' mucinous adenocarcinomas, (iii) 'microsatellite stable null' mucinous adenocarcinomas, and (iv) KRAS-mutated colorectal adenocarcinomas. Notably, hierarchical clustering analysis led to distinguish a cluster of mucinous adenocarcinomas harboring BRAF mutation and a cluster of 'microsatellite instability-high null' mucinous adenocarcinomas harboring neither BRAF mutation nor KRAS mutation. The cluster of BRAF-mutated colorectal carcinomas included almost as many mismatch repair-proficient mucinous adenocarcinomas as mismatch repairdeficient colorectal mucinous adenocarcinomas. This cluster exhibited particular clinicopathological and molecular features such as their occurrence in older patients, essentially in females, and their location in right colon. A residual polyp was rarely detected at the edges of these colorectal mucinous adenocarcinomas, but when it did, this harbored conventional adenomatous dysplasia. These mucinous adenocarcinomas frequently harbored $M L H 1$ promoter methylation in high level of mismatch repair deficiency setting or MGMT changes indicating low levels of mismatch repair deficiency and CpG island methylation phenotype. ${ }^{33,34}$ Finally, the more distinctive molecular pattern of this cluster of BRAF-mutated colorectal mucinous carcinoma was the constant low level of $\mathrm{wnt} /$ beta-catenin signaling activation in the tumor core, suggesting that BRAF mutation is mutually exclusive with wnt/ beta-catenin signaling pathway activation in colorectal mucinous adenocarcinomas. Finally, the poor disease-specific survival associated with older age at diagnosis together with $B R A F$ 
mutation regardless microsatellite instability status further justified distinguishing this cluster. Distinguishing such group of colorectal mucinous carcinomas gives to $B R A F$ mutation a role of driver in mucinous adenocarcinomas. Currently, BRAF mutation is considered as an early oncogenic driver in the so-called serrated pathway of colorectal oncogenesis for which it represents a specific marker. ${ }^{35}$ These findings lead to the conclusion that $B R A F$-mutated mucinous adenocarcinomas have the signature of serrated pathway of colorectal carcinomas. For serrated sessile adenomas with BRAF mutation destined to progress to carcinomas, the determinative event is CpG island methylation, causing bi-allelic suppression of $M L H 1$, in microsatellite instability-high carcinomas. ${ }^{31}$ The determinative events are less clear in microsatellite stable carcinomas. Notably, MGMT changes have been detected in the progression of serrated adenomas. ${ }^{36,37}$

'Microsatellite instability_high null' colorectal mucinous adenocarcinomas exhibited higher nuclear beta-catenin expression than $B R A F$-mutated colorectal mucinous adenocarcinomas. In this cluster, patients were younger and some of them had suspected or proved Lynch syndrome. Exon 3 betacatenin gene mutation was searched in patients with higher nuclear expression of beta-catenin and finally found in two patients with proved Lynch syndrome. Previous studies have indicated a relatively high frequency of pathogenic beta-catenin mutations in Lynch cancers. ${ }^{22,38}$ Like in our study, all pathogenic mutations reported were missense changes causing alterations of threonine at codon 41 or serine at codon 45 . Johnson et $a l^{22}$ have suggested that exon 3 beta-catenin mutation occur during or after progression of adenoma to carcinoma in Lynch syndrome. This cluster comprised no MLH1-deficient colorectal mucinous adenocarcinoma with MLH1 promoter methylation harboring KRAS mutation, a particular profile of $B R A F$ wild-type MSI-H colorectal carcinomas recently described in association with conventional adenomas. ${ }^{39}$ Conversely, such pattern was observed in the control group of adenocarcinoma with mucinous component. From a clinical point of view, this cluster of 'microsatellite instability-high null' mucinous adenocarcinomas is probably the most heterogeneous including unproved inherited colon cancer syndrome, Lynch-like colon cancer syndrome, and serrated pathway-associated mucinous adenocarcinomas.

Hierarchical clustering analysis identified two clusters exclusively constituted of microsatellite stable colorectal mucinous adenocarcinomas, only segregated by the detection of KRAS mutation. These two clusters shared many clinicopathological findings as well as many of the clinicopathological and demographic findings of the control group of adenocarcinomas, not other specified. These groups also reproduced many clinicopathological characteristics of the historic series of mucinous adenocarcinomas, ${ }^{3,4}$ ie, the association with contiguous villous or tubulo-villous adenomas and association with inflammatory bowel disease. Indeed, this group of mucinous adenocarcinoma was associated with contiguous polyp or at least another polyp or carcinoma elsewhere in the colon in $80 \%$ of cases. These mucinous adenocarcinomas were more frequently located in the left side and observed in male patients. However, even in bringing together these two groups of microsatellite stable colorectal adenocarcinomas, the rate of KRAS mutation remains significantly higher than in the control group of adenocarcinomas, not other specified, ( $75 \%$ vs 33\%). Higher frequency of contiguous polyp at the edges of colorectal adenocarcinomas harboring KRAS mutation has been reported. ${ }^{31}$ Interestingly, in the present study, although most of contiguous polyps were conventional adenomas arising via the APC mutation pathway, traditional serrated adenoma was sometimes observed at the edges of mucinous adenocarcinomas with KRAS mutation of the right colon, demonstrating the involvement of serrated pathway in some KRAS-mutated mucinous carcinomas. This is in accordance with a recent study reporting that $46 \%$ of traditional serrated adenomas with invasive carcinoma showed an extracellular mucinous component, lot of them harboring KRAS mutation. ${ }^{40}$

The high frequency of MGMT changes (ie, loss of MGMT protein expression and / or MGMT promoter methylation) was a frequent pattern of $K R A S$-mutated mucinous adenocarcinomas. Like others, we have observed an imperfect concordance between the loss of protein expression using immunohistochemistry and MGMT promoter methylation. ${ }^{34,41}$ For this reason, we have chosen to integrate in hierarchical clustering analysis all the MGMT changes detected regardless their translation. The poor concordance can be secondary to various causes including methodology of methylation assay and alternative molecular mechanisms that regulate MGMT expression. For example, Halford et $a l^{42}$ have described somatic missense MGMT gene mutation in colorectal carcinomas, accompanied in some cases by reduced or loss of MGMT expression. Some studies have reported that colorectal carcinomas harboring KRAS mutation was associated with particular molecular correlates including MGMT gene inactivation. In this regard, it has been suggested that MGMT inactivation leads to malignant progression of adenomas to carcinomas in combination with KRAS and TP53 mutations. ${ }^{43,44}$ Finally some authors found MGMT methylation to be associated with low level of microsatellite instability and $\mathrm{CpG}$ island methylation phenotype. ${ }^{33,45-47}$ It has been reported that inactivation of MGMT by methylation plays a role in several alternate pathways, including the KRAS-mutated traditional-serrated adenoma pathway, ${ }^{48}$ described by Jass et $a l^{49}$ as a fusion pathway.

The frequent MGMT inactivation in colorectal mucinous adenocarcinoma might open therapeutic perspectives for tumors developing naturally many 
drug resistances, ie, conventional cytotoxic chemotherapy ${ }^{27,50}$ and EFGR monoclonal antibodies. ${ }^{51,52}$ As MGMT exerts a central role in repairing alkylation-induced damage, ${ }^{18}$ MGMT inactivation in $B R A F$ and $K R A S$-mutated mucinous adenocarcinomas may indicate a high level of sensitivity to alkylating agents. It has been already documented that dacarbazine was active after failure of standard therapies in patients with metastatic colorectal carcinomas harboring inactivation of $M G M T .^{53,54}$ However, responses of patients with mucinous adenocarcinoma to chemotherapy might vary not only owing molecular characteristics of tumor but also owing the markedly deficient physical properties of tumor microenvironment, including the mucus layer and poorly developed microvasculature preventing access of drugs to tumor cells. ${ }^{55}$

In summary, mucinous adenocarcinomas belong to distinct clinicopathological clusters with epidemiological, prognostic, and therapeutic relevance. This study suggests that both KRAS and $B R A F$ mutations play a major role as driver in the multiple alternate pathways governing progression of colorectal mucinous carcinomas, whatever (epi) genetic background. Larger series are required to refine the distinct clusters of mucinous adenocarcinomas based on histomolecular features.

\section{Acknowledgments}

The study received Grant PROG/09/03 from the French 'Direction de l'Hospitalisation et l'Organisation des Soins' (DHOS).

\section{Disclosure/conflict of interest}

The authors declare no conflict of interest.

\section{References}

1 Parham D. Colloid carcinoma. Ann Surg 1923;77:90-105.

2 Hamilton SR, Bosman FT, Boffetta P, et al. Carcinoma of the colon and rectum. In: Bosman FT, Carneiro F, Hruban RH, et al. (eds). World Health Organization Classification of Tumours of the Digestive System, 4th edn. IARC Press: Lyon, France, 2010, pp 134-146.

3 Symonds DA, Vickery AL. Mucinous carcinoma of colon and rectum. Cancer 1976;37:1891-1900.

4 Umpleby HC, Ranson DL, Williamson RCN. Peculiarities of mucinous colorectal carcinoma. Br J Surg 1985;72:715-718.

5 Song GA, Deng GR, Bell I, et al. Mucinous carcinomas of the colorectum have distinct molecular genetic characteristics. Int J Oncol 2005;26:745-750.

6 Tanaka H, Deng GR, Matsuzaki K, et al. BRAF mutation, CpG island methylator phenotype and microsatellite instability occur more frequently and concordantly in mucinous than non-mucinous colorectal cancer. Int J Cancer 2006;118:2765-2771.
7 Messerini L, Vitelli F, DeVitis LR, et al. Microsatellite instability in sporadic mucinous colorectal carcinomas: relationship to clinico-pathological variables. J Pathol 1997;182:380-384.

8 Kanemitsu Y, Kato T, Hirai T, et al. Survival after curative resection for mucinous adenocarcinoma of the colorectum. Dis Colon Rectum 2003;46:160-167.

9 Alexander J, Watanabe T, Wu TT, et al. Histopathological identification of colon cancer with microsatellite instability. Am J Pathol 2001;158:527-535.

10 Greenson JK, Huang SC, Herron C, et al. Pathologic predictors of microsatellite instability in colorectal cancer. Am J Surg Pathol 2009;33:126-133.

11 Consorti F, Lorenzotti A, Midiri G, et al. Prognostic significance of mucinous carcinoma of colon and rectum: a prospective case-control study. J Surg Oncol 2000;73:70-74.

12 Farhat MH, Barada KA, Tawil AN, et al. Effect of mucin production on survival in colorectal cancer: a casecontrol study. World J Gastroenterol 2008;14:6981-6985.

13 Vogelstein B, Fearon ER, Hamilton SR, et al. Genetic alterations during colorectal tumor development. N Engl J Med 1988;319:525-532.

14 Thibodeau SN, Bren G, Schaid D. Microsatellite instability in cancer of the proximal colon. Science 1993;260:816-819.

15 Muzny DM, Bainbridge MN, Chang K, et al. Comprehensive molecular characterization of human colon and rectal cancer. Nature 2012;487:330-337.

16 Behl AS, Goddard KA, Flottemesch TJ, et al. Costeffectiveness analysis of screening for KRAS and BRAF mutations in metastatic colorectal cancer. J Natl Cancer Inst 2012;104:1785-1795.

17 Buecher B, Cacheux W, Rouleau E, et al. Role of microsatellite instability in the management of colorectal cancers. Dig Liver Dis 2013;45:441-449.

18 Pegg AE. Mammalian O6-alkylguanine-DNA alkyltransferase regulation and importance in response to alkylating carcinogenic and therapeutic agents. Cancer Res 1990;50:6119-6129.

19 Jenkins MA, Hayashi S, O’Shea A-M, et al. Pathology features in Bethesda guidelines predict colorectal cancer microsatellite instability: a population-based study. Gastroenterology 2007;133:48-56.

20 Weisenberger DJ, Siegmund KD, Campan M, et al. CpG island methylator phenotype underlies sporadic microsatellite instability and is tightly associated with BRAF mutation in colorectal cancer. Nat Genet 2006;38: 787-793.

21 Suraweera N, Duval A, Reperant M, et al. Evaluation of tumor microsatellite instability using five quasimonomorphic mononucleotide repeats and pentaplex PCR. Gastroenterology 2002;123:1804-1811.

22 Johnson V, Volikos E, Halford SE, et al. Exon $3 \beta-$ catenin mutations are specifically associated with colorectal carcinomas in hereditary non-polyposis colorectal cancer syndrome. Gut 2005;54:264-267.

23 Liu CL, Prapong W, Natkunam Y, et al. Software tools for high-throughput analysis and archiving of immunohistochemistry staining data obtained with tissue microarrays. Am J Pathol 2002;161:1557-1565.

24 Eisen MB, Spellman PT, Brown PO, et al. Cluster analysis and display of genome-wide expression patterns. Proc Natl Acad Sci USA 1998;95:14863-14868.

25 Shen L, Kondo Y, Rosner GL, et al. MGMT promoter methylation and the field defect in sporadic colorectal cancer. J Natl Cancer Inst 2005;97:1330-1338. 
26 Giovannucci E, Ogino S. DNA methylation, field effects, and colorectal cancer. J Natl Cancer Inst 2005; 97:1317-1319.

27 Negri FV, Wotherspoon A, Cunningham D, et al. Mucinous histology predicts for reduced responsiveness and survival in advanced colorectal cancer. Ann Oncol 2005;16:1305-1310.

28 Verhulst J, Ferdinande L, Demetter P, et al. Mucinous subtype as prognostic factor in colorectal cancer: a systematic review and meta-analysis. J Clin Pathol 2012;65:381-388.

29 Wolfman EF, Astler VB, Coller FA. Mucoid adenocarcinoma of the colon and rectum. Surgery 1957;42:846-852.

30 Andrici J, Farzin M, Sioson L, et al. Mismatch repair deficiency as a prognostic factor in mucinous colorectal cancer. Mod Pathol 2016;29:266-272.

31 Rosty C, Young JP, Walsh MD, et al. Colorectal carcinomas with KRAS mutation are associated with distinctive morphological and molecular features. Mod Pathol 2013;26:825-834.

32 Laurent-Puig P, Olschwang S, Delattre O, et al. Association of Ki-ras mutation with differentiation and tumor-formation pathways in colorectal carcinomas. Int J Cancer 1991;49:220-223.

33 Jass JR. Classification of colorectal cancer based on correlation of clinical, morphological and molecular features. Histopathology 2007;50:113-130.

34 Whitehall VL, Walsh MD, Young J, et al. Methylation of O-6-methylguanine DNA methyltransferase characterizes a subset of colorectal cancer with low-level DNA microsatellite instability. Cancer Res 2001;61:827-830.

35 O'Brien MJ, Yang S, Mack C, et al. Comparison of microsatellite instability, CpG island methylation phenotype, BRAF and KRAS status in serrated polyps and traditional adenomas indicates separate pathways to distinct colorectal carcinoma end points. Am J Surg Pathol 2006;30:1491-1501.

36 Oh K, Redston M, Odze RD. Support for hMLH1 and MGMT silencing as a mechanism of tumorogenesis in the hyperplastic-adenoma-carcinoma (serrated) carcinogenic pathway in the colon. Hum Pathol 2005;36: 101-111.

37 Bettington M, Walker N, Rosty C, et al. Clinicopathological and molecular features of sessile serrated adenomas with dysplasia or carcinoma. Gut 2017;66:97-106.

38 Miyaki M, Iijima T, Kimura J, et al. Frequent mutations of $\beta$-catenin and $A P C$ genes in primary colorectal tumors from patients with hereditary nonpolyposis colorectal cancer. Cancer Res 1999;59:4506-4509.

39 Farchoukh L, Kuan S-F, Dudley B, et al. MLH1deficient colorectal carcinoma with wild-type $B R A F$ and MLH1 promoter hypermethylation harbor KRAS mutations and arise from conventional adenomas. Am J Surg Pathol 2016;40:1390-1399.

40 Tsai J-H, Liau J-Y, Lin Y-L, et al. Traditional serrated adenoma has two pathways of neoplastic progression that are distinct from the sessile serrated pathway of colorectal carcinogenesis. Mod Pathol 2014;27:1375-1385.

41 Shima K, Morikawa T, Baba Y, et al. MGMT promoter methylation, loss of expression and prognosis in 855 colorectal cancers. Cancer Causes Control 2011;22:301-309.
42 Halford S, Rowan A, Sawyer E, et al. O (6)-Methylguanine methyltransferase in colorectal cancers: detection of mutations, loss of expression, and weak association with G:C > A:T transitions. Gut 2005;54:797-802.

43 Esteller M, Toyota M, Sanchez-Cespedes M, et al. Inactivation of the DNA repair gene O6-methylguanineDNA methyltransferase by promoter hypermethylation is associated with $\mathrm{G}$ to A mutations in K-ras in colorectal tumorigenesis. Cancer Res 2000;60:2368-2371.

44 Esteller M, Risques RA, Toyota M, et al. Promoter hypermethylation of the DNA repair gene $\mathrm{O}$ (6)methylguanine-DNA methyltransferase is associated with the presence of $\mathrm{G}: \mathrm{C}$ to A:T transition mutations in p53 in human colorectal tumorigenesis. Cancer Res 2001;61:4689-4692.

45 Ogino S, Kawasaki T, Kirkner GJ, et al. CpG island methylator phenotype-low (CIMP-low) in colorectal cancer: possible associations with male sex and KRAS mutations. J Mol Diagn 2006;8:582-588.

46 Nagasaka T, Sasamoto H, Notohara K, et al. Colorectal cancer with mutation in BRAF, KRAS and wild-type with respect to both oncogenes showing different patterns of DNA methylation. J Clin Oncol 2004;22:4584-4594.

47 Ogino S, Kawasaki T, Kirkner GJ, et al. Molecular correlates with MGMT promoter methylation and silencing support $\mathrm{CpG}$ island methylator phenotypelow (CIMP-low) in colorectal cancer. Gut 2007;56: 1564-1571.

48 Minoo P. Toward a molecular classification of colorectal cancer: the role of MGMT. Front Oncol 2013;3:266.

49 Jass JR, Baker K, Zlobec I, et al. Advanced colorectal polyps with the molecular and morphological features of serrated polyps and adenomas: concept of a 'fusion' pathway to colorectal cancer. Histopathology 2006;49: 121-131.

50 Catalano V, Loupakis F, Graziano F, et al. Mucinous histology predicts for poor response rate and overall survival of patients with colorectal cancer and treated with first line oxaliplatin- and/or irinotecan-based chemotherapy. Br J Cancer 2009;100:881-887.

51 Van Cutsem E, Köhne CH, Láng I, et al. Cetuximab plus irinotecan, fluorouracil, and leucovorin as first-line treatment for metastatic colorectal cancer: updated analysis of overall survival according to tumor KRAS and BRAF mutation status. J Clin Oncol 2011;29:2011-2019.

52 Douillard JY, Oliner KS, Siena S, et al. PanitumumabFOLFOX4 treatment and RAS mutations in colorectal cancer. N Engl J Med 2013;369:1023-1034.

53 Shacham-Shmueli E, Beny A, Geva R, et al. Response to temozolomide in patients with metastatic colorectal cancer with loss of MGMT expression: a new approach in the era of personalized medicine? J Clin Oncol 2011;29:262-265.

54 Amatu A, Sartore-Bianchi A, Moutinho C, et al. Promoter CpG island hypermethylation of the DNA repair enzyme MGMT predicts clinical response to dacarbazine in a phase II study for metastatic colorectal cancer. Clin Cancer Res 2013;19:2265-2272.

55 Hugen N, Brown G, Glynne-Jones R, et al. Advances in the care of patients with mucinous colorectal cancer. Nat Rev Clin Oncol 2016;13:361-369.

Supplementary Information accompanies the paper on Modern Pathology website (http://www.nature.com/ modpathol) 